\title{
Démographie médicale et techniques juridiques d'incitation
}

\author{
Anne-Sophie Ginon* \\ Maître de conférences en droit privé \\ Université de Paris Ouest Nanterre La Défense, (IRERP UMR 7029), \\ 200 avenue de la République, 92001 Nanterre Cedex
}

\section{Résumé}

Sous le vocable « mesures incitatives » prospèrent différentes techniques juridiques qui méritent aujourd'hui d'être attentivement étudiées tant la frontière devient difficile à tracer entre les mesures "d'incitation-encouragement » et les mesures " d'incitation-pénalité ». La question de la démographie de la profession médicale constitue un laboratoire privilégié pour analyser le déploiement des dispositifs juridiques, le législateur étant, depuis quelques années à la recherche des « incitants » les plus performants pour orienter les comportements d'installation des médecins exerçant en libéral.

๑) 2011 IDMP/Lavoisier SAS. Tous droits réservés

\begin{abstract}
Mots clés : Régulation démographique, professionnels de santé, médecins, mesures incitatives et coercitives, Loi HPST, contrat santé solidarité, pénalité.
\end{abstract}

\section{Abstract}

"Incentives" and demography in the medical sector. By "incentives", we mean a series devices that have been given insufficient attention in the legal sphere. The article aims at analysing the border between soft and hard incentives. Incentives can be particularly useful to deal with the issue of demography in the medical sector, and modify the behaviour of doctors when opening their medical practice. ๑ 2011 IDMP/Lavoisier SAS. Tous droits réservés

Keywords: medical demography, health's professionals, doctors, soft and hard incentives, contracts, penalty.

\footnotetext{
*Auteur correspondant : asginon@gmail.com doi:10.3166/pmp.28.13-25 @ 2011 IDMP/Lavoisier SAS. Tous droits réservés
} 
L'organisation territoriale de l'offre de soins est au cœur des débats actuels, que l'on pense aux dernières négociations conventionnelles qui ont eu lieu entre l'UNCAM et les syndicats représentatifs de médecins ou encore aux récentes dispositions qui ont été introduites, dans le code de la santé publique (CSP), par la loi portant réforme de l'hôpital et relative aux patients, à la santé et aux territoires (HPST) ${ }^{1}$.

L'idée est la suivante : pour implanter une offre de soins de premier recours pérenne, adaptée aux besoins de santé et compatible avec les attentes des professionnels de santé, les partenaires conventionnels comme le législateur ont opté, depuis quelques années déjà, pour l'énoncé de mesures incitatives dont l'objet est de susciter de nouveaux comportements de la part des professionnels de santé. La réflexion territoriale conduite dans la loi HPST a eu deux objectifs :

- créer des outils d'évaluation de l'offre de soins pour répondre aux besoins de santé

de la population et aux exigences d'efficience et d'accessibilité géographique ;

- inciter les professionnels de santé à une répartition optimale de la présence médicale pour garantir, notamment, la permanence des soins ;

Ainsi, il s'est agi de construire à partir d'une évaluation, par territoire, des besoins en implantation pour l'exercice des soins, des dispositifs « incitatifs » qui permettront à terme de modifier les comportements d'installation et d'exercice des professionnels de santé concernés. On voit ici qu'un lien indissociable est désormais noué entre évaluation et incitation. À cette fin, le législateur de 2009 a énoncé les valeurs auxquelles les soins doivent désormais répondre, en particulier les soins dits de «premier recours ». Il est précisé que "l'accès aux soins de premier recours ainsi que la prise en charge continue des malades sont définis dans le respect des exigences de proximité, qui s'apprécie en termes de distance et de temps de parcours, de qualité et de sécurité $»^{2}$. L'organisation des soins sur le territoire français doit donc être conforme à ces exigences nouvelles qui sont entendues de façon cumulative.

Le législateur fait alors jouer à ces valeurs un rôle bien particulier puisqu'elles autorisent la production des éléments de jugement nécessaires à l'appréciation de l'efficacité de l'offre de soins. Somme toute, il s'agit de mettre en place une échelle de valeurs pour situer l'offre de soins, la mettre en valeur ou à l'inverse la dévaluer.

La création de cette association entre évaluation et régulation de l'offre de soins sur le territoire des soins invite le juriste à porter une attention particulière aux outils juridiques d'organisation du couple «évaluation-régulation» ${ }^{3}$. Quels sont en effet les outils juridiques mis à la disposition de «l'organisateur» de l'offre de soins ? En somme, quels sont les dispositifs législatifs d'amélioration de la performance du système de soins ? À l'examen, on s'aperçoit que le législateur a principalement permis aux directeurs des agences régionales de santé de définir et de fixer des objectifs soit dans le cadre des schémas régionaux d'organisation sanitaire soit dans le cadre de contrats qui seront proposés à l'adhésion des professionnels de santé. Surtout, il est prévu d'associer à ces contrats et donc à la réalisation des objectifs définis des contreparties financières, telles celles envisagées pour les « contrats ayant pour objet d'améliorer la qualité des pratiques et la coordination des

\footnotetext{
${ }^{1}$ Loi n 2009-879 du 21 juillet 2009, J.O. du 22 juillet 2009, p. 12184.

${ }^{2}$ V. I'article L. 1411-11 du code de la santé publique.

${ }^{3} \mathrm{~V}$. Evaluation des politiques publiques sanitaires et sociales, Revue française des affaires sociales, $\mathrm{n}^{\circ} 1-2$ janvier-juin 2010.
} 
soins » $(\mathrm{CAPI})^{4}$ ou encore pour les 《contrats santé solidarité ${ }^{5}$. Ces outils présentent ainsi la particularité commune d'être laissés à la disposition des professionnels avec l'idée que ces derniers seront tentés de les adopter. On est ici au cœur de la logique incitative, laquelle entend, dans le respect de la liberté de chacun, favoriser l'adoption de comportements « meilleurs », en l'espèce plus performants, plus efficaces. À l'examen, on s'aperçoit que les textes qui traitent des questions démographiques regorgent de mesures « incitatives». Mais on perçoit aussi et malgré l'uniformité du vocabulaire que les techniques incitatives sont beaucoup plus variées qu'il n'y parait.

Lorsque l'on cherche à répertorier, voire à classer ces dispositifs, deux figures juridiques apparaissent : il existe en effet une série de mesures « incitatives» qui énoncent des normes d'encouragement pour influencer le comportement désiré chez le professionnel de santé, en lui attribuant des conséquences agréables. On parle fréquemment dans ce cas de mesures incitatives «positives». Mais il existe une seconde série de dispositifs incitatifs plus récents qui ont pour objet de décourager certains comportements en les rendant désagréables $(\$ 1)$. Fortement débattu dans l'enceinte parlementaire lors de l'adoption de la loi HPST en 2009, ce type de mesure incitative «plus contraignante », pour reprendre les termes retenus lors des débats, pose une série de questions nouvelles aux juristes qui invitent à réévaluer la qualification voire, la place qui a été fait aux normes juridiques incitatives au sein même de l'ordre juridique. Surtout, derrière la création de certains de ces dispositifs, on perçoit que l'on assiste plutôt à la naissance de nouvelles pénalités dont la validité et les conséquences juridiques n’ont, à nos yeux, pas été suffisamment évaluées (\$2).

\section{L'objet des dispositifs incitatifs de régulation de la démographie médicale}

Dans l'esprit du législateur, la création de mesures incitatives en matière de régulation démographique, correspond à la volonté de donner le choix aux médecins exerçant en libéral de souscrire ou de ne pas souscrire au dispositif qui leur est proposé. Il reste que l'exercice de ce choix peut être affecté de conséquences différentes selon que le dispositif se veut « positif » ou «négatif ». En somme, il faut aujourd'hui distinguer deux types de mesures incitatives, celles qui visent à encourager des comportements $(\S 1.1)$ que nous nommerons « incitation encouragement » et celles qui visent à freiner des comportements, voire à les pénaliser, à savoir des «incitations-pénalités». (\$1.2).

\section{1. "L'incitation-encouragement ", mesure positive pour promouvoir un comportement}

Le recours à la technique incitative positive vise à instaurer des mesures d'encouragement à suivre une conduite déterminée et à attacher à cette conduite des conséquences positives. Les individus bénéficiaires de l'incitation voient ainsi soit leurs comportements

\footnotetext{
${ }^{4}$ V. Décision du 9 mars 2009 de l'Union nationale des caisses d'assurance maladie relative à la création d'un contrat type d'amélioration des pratiques à destination des médecins libéraux conventionnés, $J O$ du 21 avril 2009 p. 6839.

${ }^{5}$ V. I'article L. 1434-8 du CSP.
} 
facilités soit récompensés s'ils suivent le comportement choisi par l'auteur de l'incitation'. Ainsi, derrière l'idée de «l'incitation-encouragement », se développent en fait deux figures distinctes d'incitations positives : il peut s'agir soit de faciliter un comportement en rendant son accomplissement plus avantageux, c'est le cas des aides octroyées par les collectivités territoriales aux médecins qui s'installent dans des zones sous-dotées (§1.1.1) soit d'attribuer une récompense pour les comportements «super-conformes » des professionnels de santé, telle la majoration d'honoraires issue de l'avenant conventionnel $\mathrm{n}^{\circ} 20$ adopté le 7 février $2007^{7}(\$ 1.1 .2)$.

\subsubsection{L'incitation qui rend l'installation en zone sous-dotée avantageuse}

Ces mesures incitatives sont les plus connues et les plus classiques en droit. Elles consistent à octroyer une aide, souvent fiscale ou sociale, pour que les acteurs visés accomplissent le comportement souhaité. Il s'agit par exemple, d'octroyer des exonérations fiscales aux professionnels de santé qui s'installent en zones médicalement sous-dotées. Ces aides pour les professionnels de santé sont aujourd'hui très nombreuses et leur recensement exhaustif ne présente pas un intérêt majeur ${ }^{8}$ pour la présente étude. On peut en revanche proposer de classer ces différentes mesures en fonction des avantages qu'elles offrent aux acteurs. En effet, il existe des aides financières qui peuvent consister en des exonérations de la taxe professionnelle normalement due par les médecins et les auxiliaires médicaux qui créent un cabinet dans une commune de moins de 2000 habitants ou s'installent dans les zones dites de revitalisation rurale. Mais il existe aussi des aides matérielles, telles celles développées par la loi du 23 février 2005, relative au développement des territoires ruraux, et l'adoption des deux décrets pris pour son application'. Ainsi, les professionnels en cours d'installation dans des zones déficitaires en offre de soins peuvent bénéficier d'une mise à disposition de locaux professionnels, voire de la mise à disposition d'un logement dans le cadre d'une convention tripartite conclue entre la collectivité territoriale, l'URCAM et le professionnel de santé. Il existe également des mesures d'autorisation d'exercice dérogatoires accordées par les conseils départementaux de l'Ordre des médecins pour faciliter la création de cabinet secondaire ${ }^{10}$.

À côté de ces aides nécessaires à l'accomplissement du comportement souhaité, on trouve celles qui entendent récompenser le comportement vertueux du professionnel de santé.

\footnotetext{
${ }^{6} \mathrm{~V}$. BOBBIO N. qui distingue dans l'ensemble de la catégorie générale de la technique d'encouragement, " celui de la facilitation dans le cas par exemple d'une subvention, d'une aide ou contribution financière, ou d'un crédit facilité et celui de la sanction positive, comme dans le cas de l'attribution d'un prix à un comportement "superconforme» ou d'une exemption fiscale. Le premier expédient permet de rendre moins lourd le coût de l'opération désirée en augmentant les moyens nécessaires à son accomplissement, ou en en diminuant la charge; par le second, on tente de rendre l'opération attrayante, en assurant à celui qui l'accomplit la gratification d'un avantage, ou en lui évitant un désavantage, une fois le comportement accompli », Essais de théorie du droit, Bruylant, L.G.D.J., p. 75 et s.

${ }^{7}$ V. I'arrêté du 23 mars 2007, portant approbation de l'avenant $n^{\circ} 20$ à la convention nationale des médecins généralistes et des médecins spécialistes, JO du 28 mars 2007

${ }^{8} \mathrm{~V}$. sur ce point, les documents diffusés sous la forme de tableaux et mis à disposition par les URML.

${ }^{9} \mathrm{~V}$. les décrets $n^{\circ}$ 2005-1724 et $n^{\circ}$ 2005-1728 du 30 décembre 2005.

${ }^{10} \mathrm{~V}$. le décret $\mathrm{n}^{\circ}$ 2005-481 du 17 mai 2005 modifiant le code de déontologie médicale.
} 


\subsubsection{L'incitation qui récompense le professionnel qui exerce de façon vertueuse en zone sous-dotée}

Ce système est celui qu'a choisi l'avenant conventionnel n 20 qui a été signé en février 2007. Pour favoriser l'installation et le maintien des médecins généralistes en zones déficitaires, les parties conventionnelles ont décidé de récompenser le comportement des «bons médecins » exerçant dans ces zones en leur octroyant une majoration de leurs honoraires de $20 \%$. Le mécanisme est optionnel puisque chaque médecin généraliste exerçant en libéral et s'installant ou qui est installé dans une zone déficitaire peut adhérer à l'option conventionnelle proposée, laquelle le contraint à répondre à un certain nombre de conditions liées à son exercice en contrepartie de la majoration de ses honoraires. En adhérant à l'option conventionnelle, le médecin s'engage ainsi à favoriser l'exercice groupé de son activité par le recours à des remplaçants et à participer à la permanence des soins. Il s'engage également à justifier d'une activité réalisée au $2 / 3$ auprès de patients résidant dans une zone sous-médicalisée.

Ce dispositif s'ajoute aux autres dispositifs conventionnels déjà mis en place.Aussi, les parties indiquent-elles que cette option peut se cumuler avec les deux contrats de bonnes pratiques relatifs à l'exercice des médecins généralistes en milieu rural et en zones franches urbaines, signés le 12 janvier 2005. Ces contrats prévoient en effet des indemnités supplémentaires pour les médecins qui s'engagent à assurer des soins dans les zones franches urbaines (ZFU) ainsi que pour les médecins qui exercent en milieu rural, c'est-à-dire dans un cabinet situé à plus de vingt minutes d'un service d'urgence dans un canton dont la densité en médecins généralistes par habitant est inférieure à 3 pour 5000. Au final, on constate que progressivement les partenaires conventionnels ont mis en place des mesures incitatives à adhésion individuelle pour les médecins qui souhaitent répondre aux conditions proposées par le texte conventionnel. Ces mesures offrent alors une récompense financière positive pour les médecins qui adoptent les comportements encouragés.

Il reste néanmoins à observer qu'au-delà des mesures positives dites «d'encouragement », des discussions ont récemment eu lieu sur la création de mesures plus coercitives, plus contraignantes. C'est le débat qui s'est ouvert en 2007 lors de la négociation de la convention avec les infirmières exerçant en libéral et c'est également celui qui a lieu récemment dans le cadre de l'adoption de la loi portant réforme de l'hôpital. Il s'agira ici de s'intéresser plus précisément à la mesure qui a été introduite à l'article 43 de ladite loi et dont l'objet est de « pénaliser» les mauvais comportements des médecins pour les inciter à dispenser des soins de premier recours dans les zones médicalement sous-dotées ${ }^{11}$.

\section{2. "L'incitation-pénalité ", mesure négative pour décourager certains comportements : l'exemple du " contrat santé solidarité »}

Le législateur de 2009 a souhaité construire un nouveau dispositif incitatif pour aller plus loin dans l'orientation des comportements et favoriser une meilleure répartition de l'offre de soins sur le territoire. Cette mesure dite «incitative » l'est cette fois-ci de façon

\footnotetext{
${ }^{11}$ D'autres outils de régulation démographique ont été adoptés dans cette loi, tels la réorientation des étudiants en médecins et des internes vers les régions les moins pourvues ou le contrat d'engagement de service public qui prévoit le versement aux étudiants en médecine d'une allocation pendant toute la durée de leurs études en échange d'un engagement à exercer à titre libéral ou salarié dans une zone sous-dotée.
} 
négative : le professionnel de santé qui n'accomplit pas le comportement désiré (conclure le « contrat santé solidarité », prévu à l'article L. 1434-8 du CSP) se voit appliquer une pénalité financière.

Dans le cadre de la mise en place du schéma régional d'organisation des soins qui permettra dans un délai de trois ans à compter de son entrée en vigueur de déterminer les zones dans lesquelles le niveau de l'offre de soins médicaux est particulièrement élevé, le directeur régional de l'agence régionale de santé pourra, «si «l'offre de soins de premier recours ne suffit pas à répondre aux besoins en santé de la population dans certains territoires de santé, proposer aux médecins exerçant dans les zones (sur-dotées) d'adhérer à un contrat santé solidarité par lequel ils s'engagent à contribuer à répondre aux besoins de santé de la population des zones sous dotées où les besoins en implantations ne sont pas satisfaits ». Surtout, le législateur a ajouté que «les médecins qui refusent de signer un tel contrat, ou qui ne respectent pas les obligations qu'il comporte pour eux, s'acquittent d'une contribution forfaitaire annuelle, au plus égale au plafond mensuel de la sécurité sociale». Pour mettre fin aux amendements qui tendaient à rendre cette mesure obligatoire, la ministre chargée de la santé a précisé combien elle était attachée au caractère facultatif de la mesure. Elle a ainsi indiqué qu'il s'agissait ici de prévoir une «mesure à caractère incitatif, la démarche devant être fondée sur le volontariat, c'est-à-dire qu'il doit être possible de s'exonérer par une redevance modérée du contrat santé solidarité $»^{12}$.

Est ainsi mis en place un «contrat santé solidarité » dont le législateur renvoie la définition du contenu à l'adoption d'un décret mais dont le refus de conclusion sera sanctionné par ce que les syndicats représentatifs des médecins ont très vite dénommé une «taxe Bachelot » et dont le montant maximum a été fixé à la somme de 2865 euros. Il s'agit donc de décourager les comportements non conformes des médecins exerçant en zones sur-dotées en leur demandant par le biais de la signature d'un contrat d'apporter des soins de premiers recours aux patients résidant dans les zones sous-dotées. La pénalité est forfaitaire puisqu'elle est déconnectée et indépendante de l'évaluation du préjudice subi par les personnes résidants dans les zones sous-dotées. La logique est ainsi inverse de celle retenue auparavant puisqu'il s'agit non plus d'encourager un comportement mais bien de décourager les comportements des médecins ne dispensant pas de soins dans les zones sous-dotées par la création d'une peine identique qui sanctionnera non seulement les refus de signature, mais également le manquement aux obligations contractuelles des professionnels de santé signataires de ces contrats.

Il reste à observer que le dispositif adopté marque une profonde rupture avec la logique incitative contractuelle introduite dans le droit de l'assurance maladie depuis la loi du 13 août 2004. Dans cette loi en effet, les incitations contractuelles avaient un tout autre objet. Elles consistaient à rendre favorable la conclusion de contrats dont le contenu était jugé favorable pour la réforme du système. Telle est la logique développée pour les « contrats responsables $»^{13}$. Le dispositif incitatif entend en effet peser sur la conclusion d'un certain type de contrats de couverture maladie complémentaire. À cette fin, le législateur a associé le bénéfice de nouvelles mesures d'exonérations fiscales et sociales à un contenu contractuel

\footnotetext{
${ }^{12}$ V. les débats devant le Sénat du 28 mai 2009.

${ }^{13}$ V. I'article L. 871-1 du Code de la sécurité sociale et le décret n²005-1126 du 29 septembre 2005, relatif au contenu des dispositifs d'assurance maladie complémentaire bénéficiant d'une aide et modifiant le code de la sécurité sociale.
} 
prédéterminé par la loi. L'incitation est économique et elle consiste à offrir des avantages aux souscripteurs de «contrats responsables ». On voit ici qu'en n'interdisant pas la dérogation, le législateur laisse les organismes de protection sociale complémentaire qui le souhaitent, libres de contracter d'autres types de couverture en matière de santé, même s'ils sont économiquement encouragés à proposer des «contrats responsables » à leurs assurés. À l'opposé, le nouveau dispositif proposé à l'article L. 1434-8 du CSP ne fixe pas de «prime » à la conclusion des « contrats santé solidarité » mais se propose de décourager les refus de conclusion en les pénalisant. Les contrats santé solidarité reposent ainsi sur ce qu'il est désormais convenu d'appeler des «incitations négatives».

Il faut alors à rechercher le sens de ces dispositifs incitatifs qui, s'ils obéissent à une philosophie commune, font très souvent l'objet de critiques aujourd'hui : ces dernières portent tant sur leur méthodologie que sur leur efficacité, voire dans certains cas, et c'est le point de vue que nous adopterons ici, sur leur validité.

\section{Le sens des dispositifs incitatifs de régulation de la démographie médicale}

Bien que distinctes, les techniques d'incitation examinées obéissent à une philosophie commune : il est présupposé que l'individu sera conduit à opter pour le comportement désiré. Il s'agit toujours de supposer que l'acteur ou encore, pour adopter le vocabulaire économique associé à ces mesures, que «l'agent» optera pour le comportement encouragé (Bureau, Mougeot, Studer, 2010). Les dispositifs incitatifs, quels qu'ils soient, ont ainsi en commun de reposer sur un modèle théorique identique : celui qui consiste à percevoir le médecin comme un agent qui réalise des choix rationnels parce que mû par des intérêts économiques.

\subsection{Un présupposé commun : le médecin optera pour le comportement encouragé}

Le point commun de l'ensemble des dispositifs incitatifs est d'offrir des facilités, des avantages financiers et matériels à ceux qui font le choix de s'installer ou d'exercer dans des zones sous-médicalisées. Les professionnels de santé restent donc toujours libres de ne pas opter pour le comportement désiré et donc de faire le choix de ne pas bénéficier de ces facilités ou ces avantages. La technique est, par excellence, incitative puisqu'elle préserve le libre choix des individus tout en faisant le pari que les individus, «optimisateurs » de leurs intérêts, choisiront la formule qui leur est économiquement la plus avantageuse. Il s'agit bien ici d'orienter les choix des professionnels de santé avec un présupposé individuel fort: chacun optera pour la formule qui lui conviendra le mieux à l'issue d'un calcul qui lui appartient.

Il faut alors tout de suite souligner que ces dispositifs incitatifs reposent toujours sur l'idée que les professionnels de santé ont un choix à faire, celui de leur lieu d'installation. L'assise fondamentale sur laquelle repose les dispositifs incitatifs est le principe de la liberté d'installation des professionnels de santé qui peuvent faire le choix d'un mode d'exercice libéral et exercer dans la zone qu'ils ont choisie. Le fondement des mesures incitatives est alors le suivant : c'est parce que le professionnel de santé peut s'installer dans la zone de son choix que les mesures législatives ou conventionnelles prétendent pouvoir jouer sur ce choix en posant des « incitants » pour influer et faire adopter au professionnel de santé le comportement souhaité. 
Aussi, ces « incitants » reposent-ils sur une vision des choix d'installation effectués par les professionnels de santé qui sont érigés en «modèles ». La construction juridique d'incitants oblige à prendre la mesure des comportements d'installation des professionnels, à déterminer ceux qui seraient les plus adaptés pour couvrir l'ensemble du territoire et ainsi à élire les décisions les plus favorables et les encourager financièrement. Il s'agit dès lors d'indiquer normativement le meilleur choix, de déterminer une décision de référence. Il est donc nécessaire de stabiliser le cadre de leurs choix parmi des plans optionnels. À l'examen, on observe que les références choisies reposent uniquement sur une rationalité économique puisqu'il est attendu des incitations financières, telles les majorations des honoraires ou les exonérations fiscales, qu'elles décideront les professionnels à s'installer ou à continuer d'exercer dans les zones déficitaires. Ces dispositifs ont donc vocation à convaincre le professionnel. Le professionnel de santé est ainsi mû par des intérêts, des rationalités économiques qui si elles sont modifiées, produiront des effets sur son comportement. On retrouve l'idée centrale qui traverse «l'économie standard » aujourd'hui, idée selon laquelle les individus sont exclusivement portés par des intérêts économiques qui les font agir ou ne pas agir et c'est par le jeu du calcul économique que l'on prétend rationaliser leur comportement (Le Pen, 2009). La construction du dispositif incitatif implique de repérer les préférences des médecins en leur associant une estimation économétrique pour éclairer les décideurs publics sur les outils à mettre en place (Ammi, Peyron, 2009). Les règles de droit sont ainsi figurées à travers des modifications de prix ou de coûts et l'action correctrice des comportements prend très souvent la forme d'incitation monétaire qui facilite les calculs d'optimisation (Affichard, Lyon-Caen, Vernac, 2009).

\subsection{Des techniques incitatives discutées dans leurs effets mais également dans leur validité}

Une fois les présupposés mis au jour, on peut être tenté de classer les différentes analyses qui sont actuellement proposées pour appréhender les mesures incitatives. On peut bien sûr, dans un premier temps, y voir l'expression d'un mouvement plus vaste de mutation de l'action publique, en particulier de l'intervention étatique. Le recours à la technique incitative serait l'expression d'une mutation des politiques publiques par lesquelles «l'état régulateur » chercherait moins à contraindre «qu'à fixer des « objectifs" qu'il serait souhaitable d'atteindre, des « directives » qu'il serait opportun de suivre, à formuler des « recommandations qu'il serait bon de respecter $\gg$. Indices d'une diversification des procédés de régulation, les incitations témoigneraient de la mise en place d'une dimension nouvelle de la régulation étatique d'un type propulsif, c'est-à-dire la création de politiques actives et volontaristes (Chevallier, 2005) ${ }^{14}$. Rapportée aux politiques de santé et à la maîtrise des dépenses de santé, l'introduction des mesures incitatives serait révélatrice du poids de la norme financière dans la définition de l'activité médicale et ainsi du déclin du pouvoir médical entendu comme l'autonomie de la pratique médicale. Àl'instar des enveloppes quantitatives d'actes et des références médicales opposables (RMO), les dispositifs incitatifs participeraient à la perception économique de la pratique médicale entendue désormais comme une activité de type économique, ou tout au moins susceptible de faire l'objet d'une évaluation économique (Hassenteufel, 1999, p. 55).

\footnotetext{
${ }^{14} \mathrm{~V}$; également, L'état régulateur, Revue française d'administration publique, $n^{\circ} 111,2004 / 3$, spéc. p. 476.
} 
Mais on peut aussi, et la tentation est grande, discuter de l'efficacité même des dispositifs incitatifs. On va alors proposer d'évaluer l'impact des mesures adoptées sur le comportement des professionnels de santé en établissant des différences entre leur impact à court terme, à long terme ou encore en repérant les effets d'aubaine produits par ces dispositifs ${ }^{15}$ voire analyser la pertinence économique des mesures. Dans ce dernier cas, l'appréciation portera sur l'évaluation de la viabilité des seuils financiers choisis. La majoration de $20 \%$ des honoraires est-elle suffisante pour produire le comportement souhaité ? Il s'agira alors de savoir si le niveau de l'incitation financière constitue une référence économique pertinente pour les acteurs. La logique incitative oblige en effet les producteurs du droit à interroger l'efficacité, voire l'efficience des incitants posés. Plus encore, les postulats sur lesquels reposent les incitations pourront eux-aussi être interrogés : les indicateurs de mesure des déserts médicaux sont-ils pertinents ? En ce sens, certains critiquent déjà l'indicateur qui a été retenu par le législateur pour déterminer l'existence du déséquilibre d'installation, à savoir la densité médicale, relevant que cet outil ne prend pas en compte l'exercice réel des médecins et reflète de manière imparfaite la pratique. Ainsi, par exemple, les actes réalisés, le temps accordé aux patients ou encore l'âge des praticiens ne sont pas intégrés alors qu'ils peuvent avoir un impact important sur la densité médicale (Berland, 2005, p. 21) (Rousset, 2009, p. 201).

On peut aussi décider d'étudier la cohérence des dispositifs incitatifs au sein d'un ensemble plus vaste de mesures incitatives, voire au sein des politiques de maîtrise des dépenses de santé. Parce que les incitations traduisent un choix d'instruments parmi d'autres possibles, il est possible de discuter les choix qu'elles expriment. N'avait-on pas la possibilité d'opter pour d'autres outils plus performants? Dans un contexte d'allocation limitée des ressources, les dépenses incitatives sont en effet perçues comme des non-dépenses sur un autre bien, sur un autre service, sur un autre projet et l'on en vient à s'interroger sur les usages alternatifs des ressources financières qu'elles nécessitent. N'y a-t-il pas un paradoxe à augmenter les tarifs des honoraires de $20 \%$ quand on cherche par tous les moyens aujourd' hui à diminuer les coûts des prestations de soins? (Batifoulier, Gadreau, Vacarie, 2008) (Pellet, 2009). Le calcul économique qui est au fondement de la technique incitative est ainsi discuté dans son postulat philosophique de base : il n'y a pas de comportement plus anti-éthique que de disposer de ressources de la collectivité sans s'interroger en conscience, ne serait-ce qu'un moment, sur les usages alternatifs qu'on pourrait en faire (Le Pen, 2009). Enfin, des analyses critiques plus théoriques apparaissent sur le modèle anthropologique même sur lequel ces mesures financières reposent, à savoir le modèle de l'homo-œconomicus qui est sous-jacent à l'adoption de ces différentes mesures et qui pénètre aujourd'hui plus largement l'ensemble du domaine de l'assurance maladie (Vacarie, Allouache, Ginon, Ferkane et Leroy, 2008)

Du point de vue du juriste, il existe une autre interrogation délicate pour la viabilité même de ces dispositifs : celle qui consiste à interroger la validité de ces règles qui prennent

\footnotetext{
${ }^{15}$.On trouve sur ce point de nombreuses analyses menées par le DREES et l'IRDES ; V. par exemple, les travaux conduits par BOURGUEIL Y., MOUSQUES J. et TAJAHMADI A., Améliorer la répartition démographique des professionnels de santé: les enseignement de la littérature, Questions d'économie de la santé, $n^{\circ} 122$, mai 2007 ou ceux conduits par ATTAL K. et VANDERSCHELDEN M., La démographie à l'horizon 2030: de nouvelles projections nationales et régionales, Etudes et résultats, $n^{\circ}$ 679, fév. 2009 ou encore les résultats d'une enquête sur les carrières des médecins généralistes: les inégalités entre générations : DORMONT B. et SAMSON A.-L. Doc. de travail DREES, nº 75, janv. 2008.
} 
la forme d'incitations monétaires. Ainsi, bien avant d'étudier, voire d'évaluer les effets des mesures incitatives, faut-il s'assurer que ces mesures seront à même de produire des effets juridiques, c'est-à-dire d'être légalement admises au sein de l'ordre juridique (Jornet, 2009 , p. 112). En effet, la tentation est grande en droit de voir dans certains des dispositifs proposés non pas des incitations mais des pénalités et, c'est finalement, la qualification juridique d'incitation qui a été conférée à ces dispositifs qui mérite, selon nous, d'être discutée. Avec l'adoption de mesures incitatives négatives, le législateur pourrait avoir crée de véritables pénalités, lesquelles pour être admises dans l'ordre du droit, doivent nécessairement répondre à un certain nombre conditions. Il faut en effet se demander s'il est possible, comme le prévoit le dispositif du «contrat santé solidarité » de sanctionner l'exercice d'une liberté contractuelle, laquelle a pour objet de permettre, comme toute liberté, d'agir ou de ne pas agir.

Soucieux du respect de la hiérarchie des normes et des principes fondamentaux de notre droit, parmi lesquels le Conseil constitutionnel a érigé la liberté contractuelle en principe à valeur constitutionnelle ${ }^{16}$, le juriste est ainsi conduit à interroger la constitutionnalité de l'incitation-pénalité construite par la loi HPST. Et plus généralement, il est invité à se demander s'il existe une place dans notre droit pour la mise en place de sanctions financières dans l'exercice même d'une liberté contractuelle.

La mesure proposée à l'article L. 1434-8 du CSP constitue à notre sens un tournant dans la philosophie des règles dites « incitatives ». Il ne s'agit plus de récompenser le comportement vertueux de l'acteur en rendant son choix avantageux mais bien de pénaliser directement le choix qu'il effectue pour lui indiquer que ce choix n'est pas conforme au comportement choisi et même prôné par le législateur. Dans le contrat santé solidarité c'est en effet le refus de signature du contrat qui est pénalisé par le versement d'une amende forfaitaire. On st ainsi face à une véritable sanction pécuniaire puisque c'est la nature même du choix exercé par le professionnel de santé qui constitue le critère d'application de la charge financière supplémentaire.

La question qui vient immédiatement à l'esprit est celle de savoir si l'on peut encore considérer que l'on est face à une norme incitative de découragement. On peut légitimement en douter. Le législateur sanctionne ici directement l'exercice d'une faculté contractuelle. Et l'on peut tout simplement se demander si une telle mesure ne constituerait pas, si le Conseil constitutionnel avait été interrogé sur ce point, une atteinte disproportionnée au principe à valeur constitutionnelle de liberté de contracter, atteinte qui conduirait à nier l'existence même de cette liberté.

Dans une décision rendue en 2000, le Conseil constitutionnel a eu l'occasion de se prononcer sur une mesure qu'il a qualifiée d' «incitative » et donc il a admis la validité juridique. En l'espèce, les requérants critiquaient une contribution nouvelle applicable aux entreprises pharmaceutiques qui s'abstiendraient de conclure une convention de modération du prix de certains médicaments avec le comité économique des produits de santé. Cette imposition pouvait atteindre $70 \%$ du chiffre d'affaires et rendait pour les requérants en quelque sorte « obligatoire» la conclusion d'un contrat avec ce comité. Le Conseil va proposer un raisonnement important sur les atteintes que les incitations peuvent porter à l'exercice de

\footnotetext{
${ }^{16}$ V. Conseil constitutionnel, Décision $\mathrm{n}^{\circ}$ 2000-437 DC du 19 décembre 2000, Loi relative au financement de la sécurité sociale.
} 
la liberté contractuelle. Aussi, va-t-il indiquer que "s'il est vrai que le dispositif institué par le législateur a notamment pour finalité d'inciter les entreprises pharmaceutiques à conclure avec le comité économique des produits de santé des conventions relatives à un ou plusieurs médicament, visant la modération de l'évolution du prix de ces médicaments et à la maîtrise du coût de leur promotion, une telle incitation, inspirée par des motifs d'intérêt général, n'apporte pas à la liberté contractuelle qui découle de l'article 4 de la Déclaration des droits de l'homme et du citoyen une atteinte contraire à la Constitution».

Si l'apport de la décision est important en ce qu'il est permis de considérer que les mesures incitatives visant à influencer certains agissements, telle en l'espèce la conclusion de contrats, peuvent être admises par le Conseil constitutionnel, il reste néanmoins à observer que la mesure qui était discutée en l'espèce était une mesure incitative du type « norme d'encouragement ». Il s'agissait comme le fait d'ailleurs la législation sur les «contrats responsables » de déclencher des effets positifs ou des effets négatifs à la suite de la conclusion du contrat. L'incitation entendait ainsi jouer sur les motifs de conclusion des contrats en rendant leur conclusion avantageuse pour les «contrats responsables » ou dans le cas des entreprises pharmaceutiques moins avantageuse pour les parties non signataires.

Le dispositif du «contrat santé solidarité » issu de la loi HPST propose, quant à lui, une construction différente. S'il s'agit de rendre le refus de contracter désavantageux pour le médecin, le désavantage ne résulte pas de l'exercice de la liberté contractuelle mais lui est en quelque sorte attaché. C'est le refus de signer le contrat qui déclenche automatiquement l'application de la pénalité. La mesure aurait été tout autre si le législateur avait attaché à la non conclusion du contrat des conséquences désavantageuses par rapport à la conclusion du même contrat, telle l'impossibilité de bénéficier d'avantages sociaux et fiscaux liés à la conclusion du contrat. La frontière à tracer entre ce qui relève d'une mesure de découragement à l'adoption d'un comportement non désiré et ce qui constitue une pénalité qui sanctionne l'exercice même de la décision de comportement est alors bien mince. Elle reste néanmoins, à nos yeux, importante pour que ne soient pas mêlées sous le vocable «mesures incitatives » des techniques juridiques qui n'appartiennent pas à cette catégorie juridique de norme.

Si depuis la création du dispositif du médecin traitant, certains ont pu s'interroger sur l'effectivité du choix dont dispose l'assuré social (est-il encore possible de choisir de ne pas consulter son médecin traitant lorsque l'on est pas en mesure de prendre en charge la pénalité forfaitaire qui lui est associée ?), l'interrogation à laquelle conduit la création $\mathrm{du}$ «contrat santé solidarité » est encore différente : est-il encore possible de croire qu'il existe une liberté là où le législateur sanctionne simplement son exercice pur et simple ?

On observera qu'à propos du dispositif du médecin traitant, le Conseil constitutionnel s'est aménagé un pouvoir d'appréciation sur le niveau de la pénalité qui s'imposerait aux assurés qui n'aurait pas choisi de médecin traitant, rappelant que cette part qui restait à la charge de l'assuré devait rester compatible avec le principe de protection de la santé17. Nous ne doutons pas que si les juges devaient être saisis de la légalité de l'application d'une telle sanction qu'une question préalable de constitutionnalité soit soulevée de façon à ce que le juge constitutionnel puisse se prononcer sur le niveau de l'atteinte que la sanction porte à

\footnotetext{
${ }^{17}$ Conseil constitutionnel, Décision n²004-504 DC, Loi portant réforme de l'assurance maladie, JO du 17 août 2004, p. 14670.
} 
l'exercice même de la liberté contractuelle. Il pourra à tout le moins s'attacher à examiner si ce niveau d'atteinte, au nom d'un motif d'intérêt général, n'est pas tel qu'il en revient à nier l'existence la liberté de contracter. Et ce sont ainsi les contentieux à venir qui nous dirons si le législateur a opté en la matière pour l'outil juridique le plus performant... au sens le plus fort en droit, à savoir pour un outil juridique qui répond aux conditions de validité exigées pour une insertion légale dans notre système juridique.

\section{Conclusion}

Si les dispositifs incitatifs ont aujourd'hui la faveur du législateur pour réorganiser territorialement l'offre de soins, leur analyse juridique conduit à poser une question préalable à celle souvent posée de leur efficience. La qualification « d'incitation » suffit-elle pour que le dispositif puisse légalement s'insérer dans l'ordre juridique ? À l'heure où les politiques publiques recourent de plus en plus à ce qu'il est convenu d'appeler «des procédés plus souples de régulation », la question n'est à ce jour pas réglée sur le plan du droit dès lors que dans leur technique même ces dispositifs entendent s'appuyer sur une liberté fondamentale des acteurs qui est la liberté contractuelle, voire dans certains cas la liberté individuelle de chacun.

\section{Bibliographie}

AFFICHARD J, LYON-CAEN A. et VERNAC S., (2009), De l'analyse économique à l'évaluation du droit du travail. Quelques leçons d'un programme de recherche, Revue de droit du travail 11, 631-638.

AMMI M. et PEYRON C., (2009), Incitations à l'offre de prévention et préférences en médecine générale : l'apport de la méthode DCE, Économie publique 24-25, 129-155.

BATIFOULIER P., GADREAU M., VACARIE I., (2008), , Rapport final Mire - Drees, Paris.

BERLAND Y., (2005), Rapport de la Commission démographie médicale remis au Ministre Philippe Douste-Blasy. BOBBIO N., (2000), Essais de théorie générale du droit, Bruylant L.G.D.J, Paris.

BOURGEUIL Y., MOUSQUES J. et TAJAHMADI A., (2006), Améliorer la répartition démographique des professionnels de santé : les enseignements de la littérature, Questions d'économie de la santé 116.

BOURGEUIL Y., MOUSQUES J. et TAJAHMADI A., (2007), Améliorer la répartition géographie des médecins : les mesures adoptées en France, Questions d'économie de la santé 122.

BURDILLAT M., (2009), Les nouvelles questions posées à la démographie médicale, Regards croisés sur l'économie 1 (5), 74-85.

BUREAU D., MOUGEOT M., STUDER N., (2010), Mesurer la performance de la gestions publique à la lumière de l'analyse économique, Revue française des Affaires sociales 1-2, 89-104.

CHEVALLIER J., (2005) Politiques publiques et changement social, Revue française d'administration publique $115,383-390$.

CHEVALLIER J., (2004), L'état régulateur, Revue française d'administration publique 111, 473-482.

GINON A.S., (2005), Le médecin traitant, révélateur des nouvelles fonctions de la protection sociale complémentaire, Revue de droit sanitaire et sociale 6, 907-918.

GINON A.S., (2009), Le jeu du conventionnement : effets paradoxaux et difficultés juridiques, Journal d'économie médicale 27 (4),211-221. 
HASSENTEUFELP.,(1999), Vers le déclin du « pouvoir médical »? Un éclairage européen : France, Allemagne, Grande-Bretagne, Pouvoirs 89, 51-64.

JOHANET G., (2008), L'échec de la régulation, Les tribunes de la santé 20, 97-110.

JORNET F., (2009), Le droit applicable à l'installation des professionnels de santé libéraux sur un territoire, Revue de droit sanitaire et social Hors série, .108-115.

LE PEN C., (2009), Calcul économique et décision en santé in Bras P.-L., de Pouvourville G. et Tabuteau D., Traité d'économie et de gestion de la santé, Presses de sciences politiques, Paris, 63-73.

La loi «Hôpital, patients, santé et territoires », (2009), Dossier, Revue de droit sanitaire et social 5.

La réforme du système de santé, (2009), Actualité juridique de droit administratif 40, p. 2205.

PELLET R., (2009), Assurance maladie et territoires, Revue de droit sanitaire et social, 38-64.

ROUSSET G., (2009), Entre incitation et coercition, vers la mise en place de nouveaux dispositifs de régulation de la démographie médicale in Couty E., Kouchner C., Laude A. et Tabuteau D., La loi HPST. Regards sur la réforme du système de santé, Presses de l'EHESP, Paris.

VACARIE I., (2005), Les tensions entre le droit de la santé et le droit de la sécurité sociale, Revue de droit sanitaire et social 6, 899-906.

VACARIE I., ALLOUACHE A., GINON A.S., FERKANE Y. et LEROY S., (2008), Crise de l'Etat providence ou crise de la régulation économique ? Les leçons des réformes de l'assurance maladie, Droit social, 1103-1114.

VILLENEUVE P., (2009), Une nouvelle approche territoriale de l'offre de soins ? À propos de la loi du 21 juillet 2009, La Semaine Juridique éd. G. 37, 210. 
